\title{
Eucalyptus camaldulensis DEHNH PRODUCTIVIDAD EN CULTIVOS ENERGÉTICOS BAJO RIEGO
}

\author{
Bustamante, J. A. ${ }^{3} ;$ Pérez, S. A ${ }^{1}$. y Llera, J. ${ }^{1}$
}

\section{RESUMEN}

La matriz energética de Argentina es altamente dependiente de combustibles fósiles, fundamentalmente petróleo y gas, y el país se ha convertido en lo últimos años en un neto importador de energía. Actualmente se encuentra en vigencia legislación que promueve el desarrollo de diversas fuentes de energías renovables a fin de modificar esta situación, entre ellas la biomasa en general y la biomasa forestal en particular.

Algunas especies del género Eucalyptus han sido utilizadas en diversos lugares del mundo en la implantación de cultivos energéticos debido a que en general proveen grandes cantidades de biomasa en cortos períodos de tiempo y poseen una serie de características deseables para ser usadas con este fin.

La región centro-oeste del país presenta un clima desértico donde la agricultura es posible solo mediante la aplicación de riego y donde cada vez mayor cantidad de agua de los ríos es demandada y utilizada por la población.

En Mendoza las aguas provenientes de efluentes domiciliarios tratados son utilizadas para regar áreas denominadas ACRES (Áreas de Cultivos Restringidos Especiales) en las cuales solo están permitidos cultivos que no conlleven peligro a la salud de la población.

Una de las alternativas para lograr el doble objetivo, de alcanzar una deseable diversificación de la matriz energética y un uso racional y ambientalmente aceptable del recurso hídrico que supone el agua proveniente de efluentes domiciliarios, es la implantación de cultivos energéticos con especies forestales para obtener biomasa y posteriormente transformarla en energía.

Con el objeto indicado, se instaló en la provincia de Mendoza un cultivo de Eucalyptus camaldulensis Dehnh. con densidades de 10.000 y 20.000 plantas/ha, regado con agua de efluentes domiciliarios. El diseño estadístico fue de bloques al azar, con tres repeticiones para cada densidad.

Todas las plantas del ensayo se cortaron a $15 \mathrm{~cm}$ del suelo luego del primer año de plantación y dos años después se procedió a cortar todos los brotes producidos por esas mismas plantas. El peso promedio individual de cada planta en ese primer ciclo de corta fue de $6,38 \mathrm{~kg}$, para una densidad de 10.000 plantas/ha y de $4,74 \mathrm{~kg}$ para una densidad de 20.000 plantas/ha.

Palabras clave: Eucalyptus camaldulensis, Cultivos energéticos, Riego, Efluentes Domiciliarios.

\footnotetext{
3 Docentes, investigadores, Facultad de Ciencias Agrarias, Universidad Nacional de Cuyo. MendozaRepública Argentina. jbustamante@fca.uncu.edu.ar; sperez@fca.uncu.edu.ar; ;jllera@fca.uncu.edu.ar
} 


\section{SUMMARY}

Argentina has an energy matrix highly dependent on fossil fuels, mainly oil and gas, also in recent years the country has become a net importer of energy.

There is currently legislation that promotes the development of various renewable energy sources as a way to change this situation, including biomass in general and in particular forest biomass.

Some species of the Eucalyptus genus have been used in different parts of the world in the implementation of woody energy crops, as they generally provide large amounts of biomass in short periods of time and also have a number of desirable characteristics for its use on this purpose.

In another aspect, the central-west of the country has a desert climate where agriculture is only possible through the application of irrigation, and where more and more water from rivers is demanded and used by the population.

In Mendoza, the wastewaters are conducted to areas called ACRES (Restricted Areas of Special Crops) in which only crops which present no risk for the health of the population are allowed.

For these reasons it was installed in the province of Mendoza, a Eucalyptus camaldulensis Dehnh. culture at densities of 10,000 and 20,000 plants/ha, irrigated with wastewater. The design was randomized block with three replicates for each density.

After the first year of planting, all plants were cut to $15 \mathrm{~cm}$ from soil level and two years later all shoots produced were cut. The average weight of individual plant was $6.38 \mathrm{~kg}$ for a density of 10,000 plants/ha and $4.74 \mathrm{~kg}$ for a density of 20,000 plants/ha.

Keywords: Eucalyptus camaldulensis, Energy Crops, Irrigation, Wastewater 


\section{INTRODUCCIÓN}

Argentina tiene una matriz energética altamente dependiente de hidrocarburos fósiles, fundamentalmente petróleo y gas, habiéndose transformado además en lo últimos años de un país exportador de energía en uno netamente importador.

A fin de atenuar esta circunstancia, actualmente se encuentra en vigencia legislación nacional que promueve el desarrollo de diversas fuentes de energías renovables, entre ellas la biomasa en general y la biomasa forestal en particular.

En otro aspecto, la región centro-oeste del país presenta en general un clima desértico; Mendoza concretamente se caracteriza por precipitaciones que van desde menos de $200 \mathrm{~mm}$ hasta algo más de $300 \mathrm{~mm}$ anuales, siendo la agricultura solo posible mediante la aplicación de riego, y donde cada vez mayor cantidad de agua de los ríos es demandada y utilizada por la población, cercana a los dos millones de personas, y por sus numerosas industrias.

Estos usos generan cada vez mayores volúmenes de efluentes, llegando a tal punto que se estima que la superficie que potencialmente podrían regar estos efluentes suma ya más de 12.000 ha.

En la provincia de Mendoza, las aguas provenientes de efluentes domiciliarios tratados son utilizadas para regar áreas denominadas ACRES (Áreas de Cultivos Restringidos Especiales) en las cuales solo están permitidos cultivos que no conlleven peligro a la salud de la población por su consumo.

Una de las alternativas para lograr el doble objetivo, de alcanzar una deseable diversificación de la matriz energética regional y nacional y un uso racional y ambientalmente aceptable del recurso hídrico que supone el agua proveniente de efluentes domiciliarios, es la implantación de cultivos energéticos con especies forestales para obtener biomasa y la posterior transformación de esta en energía.

Bajo los términos de cultivos energéticos; SRF (Short Rotation Forest) o SRFC (Short Rotation Forest Coppice) o SRWC (Short Rotation Woody Crops), se engloba una serie de situaciones muy diversas y en muchos casos difíciles de encuadrar.

Esto teniendo en cuenta que salvo su principal objetivo, que es la obtención de grandes volúmenes de madera en cortos tiempos de rotación, destinando en última instancia esta producción en numerosos casos a la obtención de energía, las demás características manifiestan límites bastante amplios. Así por ejemplo, en cuanto al turno de corta puede ser variable de uno a quince años, las densidades consideradas por diversos autores van desde 500 (a veces menos) a más de 100.000 plantas/ha y las especies utilizadas y sistemas de manejo son en ocasiones muy diferentes.

Algunas especies del género Eucalyptus han sido utilizadas en diversos lugares del mundo, en la implantación de cultivos energéticos con especies forestales, ya que en general proveen grandes cantidades de biomasa en cortos períodos de tiempo y poseen además una serie de características deseables para ser usadas con este fin, entre las que es posible mencionar el rápido crecimiento en su fase juvenil, la buena capacidad de rebrote, la producción de grandes cantidades de biomasa en peso seco con alto poder calórico y buena calidad como biocombustible, y su adaptación a diferentes con buena resistencia a estrés biótico y abiótico.

\section{OBJETIVO}

Evaluar el comportamiento y la productividad, en términos de peso de biomasa fresca y seca producida por hectárea, de Eucalyptus camaldulensis Dehnh. en condiciones de altas densidades de cultivo y turnos cortos de aprovechamiento, bajo condiciones de riego con agua proveniente de efluentes domiciliarios. 
El logro de este objetivo contribuirá a obtener conocimientos a nivel regional sobre la posibilidad de cultivar especies forestales en la modalidad de cultivos energéticos, con el fin de obtener biomasa para el posterior uso directo o su eventual transformación a biocombustible, brindando así una alternativa de solución a los problemas planteados precedentemente, el eficiente, racional y seguro uso de aguas provenientes de efluentes domiciliarios e industriales y la reducción de la marcada dependencia de los combustibles fósiles que presenta la matriz energética nacional. Como aspiración complementaria y simultánea, se pretende lograr que el desarrollo de esta nueva actividad sea sustentable desde el punto de vista ambiental, económico y social.

\section{ANTECEDENTES}

A nivel mundial se puede encontrar una cantidad de información sobre el uso de especies del género Eucalyptus en condiciones de alta densidad y cortas rotaciones.

Densidades de plantación de 500, 1.000, 2.000 y 4.000 árboles/ha en Eucalyptus globulus y Eucalyptus occidentalis, con turno de corta de 3 años (Sochacki et al., 2007).

Densidades de 1.937 a 2.787 árboles/ha', en Eucalyptus gunnii con cortas anuales (Forrest y Moore, 2008).

Densidad de 3.470 árboles/ha en Eucalyptus globulus, Eucalyptus nitens, Eucalyptus saligna y Eucalyptus ovata, con turnos de corta de 3 a 5 años (Senelwa y Sims, 1999).

Densidad de 4.167 árboles/ha en Eucalyptus botryoides, Eucalyptus globulus $y$ Eucalyptus ovata, con turno de corta 3 años (Guo et al., 2006).

Densidades de 1.333 a 3.700 árboles/ha en Eucalyptus nitens, Eucalyptus gunnii y Eucalyptus gunnii x Eucalyptus dalrympleana, con turnos de corta de 8 a 10 años (Purse y Richardson, 2001).

Densidades de 40.000 árboles/ha en Eucalyptus globulus y Eucalyptus camaldulensis y otro ensayo con Eucalyptus camaldulensis a 10.000 y 20.000 árboles/ha, con turnos de corta variables, desde anuales (Luger, 1999).

A nivel local, existe información que ya en la década de 1950, en Mendoza se cultivaban Eucalyptus camaldulensis y Eucalyptus tereticornis, en una densidad de 5.000 plantas/ha con turnos de 4 a 5 años, siendo el principal objetivo la obtención de postes, varillas y rodrigones para viñedos además de suministrar leña y carbón.

Posteriormente Arreghini et al. (1997) y Calderón y Bustamante (2003) llevaron a cabo ensayos para evaluar el comportamiento en vivero y en plantaciones de una serie de eucaliptos, destacándose Eucalyptus camaldulensis como uno de los más adaptados a las condiciones de sitio y clima de la zona de Mendoza.

Actualmente en la provincia de Mendoza esta especie tiene una demanda concentrada principalmente en aquellos establecimientos agropecuarios, especialmente bodegas o industrias que tienen la obligación de realizar la deposición de los efluentes líquidos que ellas generan en zonas de cultivo restringidas y sin posibilidades de ser volcados nuevamente a cauces de riego.

\section{METODOLOGÍA}

\section{Descripción y Diseño del Ensayo}

Las parcelas experimentales fueron instaladas en una propiedad rural que posee riego con aguas de afluentes domiciliarios tratados, ubicada en una de las ACRE que posee la provincia, a aproximadamente $20 \mathrm{~km}$ al norte de la ciudad capital de Mendoza. 
El material vegetal utilizado fue plantines de Eucalyptus camaldulensis Dehnh, obtenidos por semillas de procedencia local, de aproximadamente $40 \mathrm{~cm}$ de altura, criados en envases de polietileno negro, los que se retiraron al momento de la plantación, manteniendo el pan de tierra.

Dichos plantines fueron plantados en dos marcos de plantación distinta:

En una doble hilera, con distancia de $75 \mathrm{~cm}$ entre hileras, luego a $150 \mathrm{~cm}$ otra doble hilera, y así alternativamente, con un distanciamiento entre plantas de $90 \mathrm{~cm}$ en la hilera, obteniendo una densidad de 10.000 plantas por hectárea (Figura $\mathrm{N}^{\circ} 1$ ).

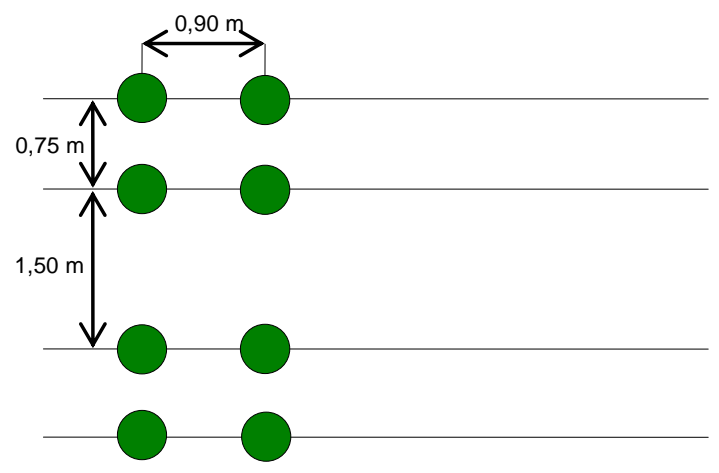

Figura $\mathbf{N}^{\circ} 1$

CROQUIS DE PLANTACIÓN

DENSIDAD 10.000 PLANTAS/ha

En una doble hilera, con distancia de $60 \mathrm{~cm}$ entre hileras, luego a $120 \mathrm{~cm}$, otra doble hilera, y así alternativamente, con un distanciamiento entre plantas de $55 \mathrm{~cm}$ en la hilera, obteniendo una densidad de 20.000 plantas/ha (Figura $\mathrm{N}^{\circ} 2$ ).

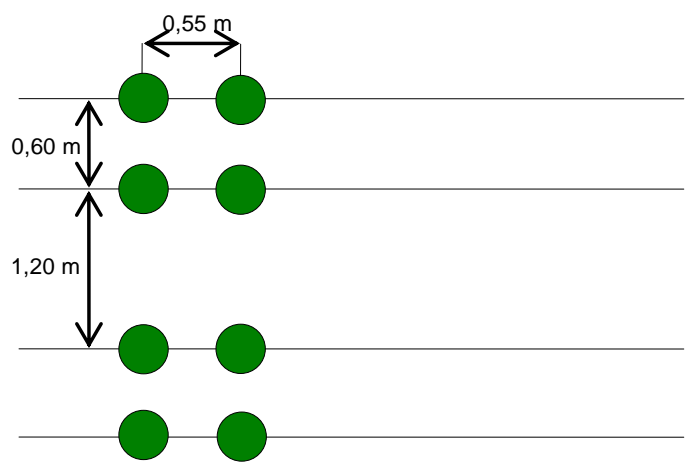

Figura $\mathrm{N}^{\circ} 2$

CROQUIS DE PLANTACIÓN

DENSIDAD 20.000 PLANTAS/ha

Se utilizó un diseño de bloques al azar con 3 replicaciones para cada una de las densidades propuestas, siguiendo en general las prescripciones de la Forestry Commission UK (Tubby y Armstrong, 2002). 
Las parcelas individuales tienen $9 \mathrm{~m} \times 11,5 \mathrm{~m}$ y $5,5 \mathrm{~m} \times 9 \mathrm{~m}$, en las densidades de 10.000 y 20.000 plantas/ha, respectivamente y contienen 10 hileras con 10 árboles cada una.

parcela.

Cada parcela posee hileras de aislación, dejando para la toma de datos 20 plantas por

\section{Instalación del Ensayo}

En agosto de 2006, sobre el sitio seleccionado se realizó una pasada de arado en forma cruzada sobre el terreno con un tractor agrícola, llegando a una profundidad aproximada de $35 \mathrm{~cm}$, a fin de desmenuzar y airear el suelo, y luego dos pasadas de rastra de discos también en forma cruzada.

Luego de la preparación del terreno e inmediatamente antes de plantación se aplicó Pendimetalin (Herbadox), emulsión al 33\%, como herbicida de preemergencia a una dosis de 4 L/ha, con cobertura total del suelo, y glifosato a razón de $4 \mathrm{~L} / \mathrm{ha}$, en los bordes de las acequias de riego para combatir la proliferación de malezas en ese sector.

Los deshierbes posteriores en el transcurso del primer año fueron realizados manualmente con azadas.

La plantación se efectuó en forma manual, colocando las plantas de eucaliptos en el fondo del surco, para así favorecer el riego de las mismas y al mismo tiempo evitar la acumulación de sales (presentes en el sitio) que se produce normalmente en la parte superior de los surcos.

Ante la presencia de hormigas negras cortadoras (Acromyrmex spp.), se llevó a cabo en forma continua y sistemática desde el mismo momento de la plantación su control, aplicándose Clorpirinfos polvo al $3 \%$ sobre las bocas de los hormigueros, una vez que eran detectados.

La disponibilidad de agua de riego en la propiedad está sujeta a lo que en Mendoza se denomina "turno de riego", que consiste en otorgar un cierto número de horas de agua en relación a la superficie de la propiedad y normalmente ese "turno" se repite con una cierta periodicidad de días.

Dada esta limitación a la disponibilidad de agua, una misma superficie tiene posibilidades de ser regada en períodos variables a lo largo del año dependiendo de las horas de turno y el caudal entregado en esas horas.

Considerando lo anterior y la información proporcionada por los trabajos de Riu y Settepani (2004) y Gutierrez y Ares (1993) sobre requerimientos de riego, se decidió efectuar los riegos aproximadamente cada 20 días desde el momento de la plantación (fines de agosto) y hasta octubre, luego cada 15 días hasta mediados de marzo y a partir de allí una vez por mes hasta mediados de junio, retomándose los mismos a partir de mediados de agosto del año siguiente.

Se efectuó dos escardas manuales con azada, para controlar el desarrollo de las malezas, una en diciembre y otra en febrero, a pesar de esto y debido a las características del agua, y el recorrido que hace la misma hasta llegar a la parcela (recorre muchos kilómetros por canales y acequias donde recibe un gran aporte de semillas de diversas malezas, muchas de ellas muy agresivas), se llegó al mes de marzo con una importante cantidad de malas hierbas, que en algunos casos representaron una seria competencia para las plantas.

Al cabo del primer período de crecimiento vegetativo, se cortó todos los fustes de los árboles existentes en cada una de las parcelas a $10 \mathrm{~cm}$ de la superficie del suelo.

Con posterioridad se dejó crecer libremente los fustes producidos en cada una de las plantas rebrotadas por un período de tiempo de dos años, realizando solo los riegos y control de malezas adecuados para el normal desarrollo del cultivo. 


\section{Toma de Datos al Primer Ciclo de Corta}

Al cabo del primer ciclo de corta (tres años desde plantación; dos años desde el rebrote), nuevamente se cortaron los fustes producidos por todas las plantas presentes en la parcela, a 15 $\mathrm{cm}$ de la superficie del suelo.

Se pesó in situ todos los fustes producidos por 60 plantas (20 plantas en cada una de las tres repeticiones) para así obtener el peso fresco individual de cada fuste, el total de cada planta y el de cada parcela.

A los datos parcelarios de peso fresco obtenidos se les aplicó el análisis de la varianza y, en caso de ser estadísticamente significativa $(p<0,05)$, se realizó una prueba de Tukey para comparar las medias de tratamientos.

Las correlaciones fueron determinadas por medio de análisis de regresión y correlación lineal simple.

Se tomó sub muestras (una planta completa de cada diez) del material vegetal extraído y se lo llevó a estufa a temperatura de $105^{\circ} \mathrm{C}$ hasta peso constante, a fin de determinar el peso seco por planta, posteriormente ese valor se usó para (por extrapolación) determinar el peso seco por parcela y luego para expresar en $\mathrm{kg}$ y $\mathrm{t}$ la materia seca producida por hectárea.

\section{RESULTADOS}

\section{Peso Fresco}

La producción de peso fresco (kg/planta) con densidad de plantación de 10.000 plantas/ha, fue de $6,38 \pm 2,32 \mathrm{~kg} /$ planta. En cambio para la densidad de 20.000 plantas/ha, fue de $4,74 \pm 1,73 \mathrm{~kg} /$ planta.

Puede advertirse que los intervalos dados por los valores mínimos y máximos para cada densidad se sobreponen entre ambos (Cuadro $\mathrm{N}^{\circ} 1$ ).

\section{PESO FRESCO POR PLANTA, PROMEDIO, MÍNIMO Y MÁXIMO SEGÚN DENSIDAD}

\begin{tabular}{|c|c|c|c|}
\hline \multirow{2}{*}{$\begin{array}{c}\text { Densidad } \\
\text { (plantas/ha) }\end{array}$} & \multicolumn{3}{|c|}{$\begin{array}{c}\text { Peso Fresco por Planta } \\
\text { (kg) }\end{array}$} \\
\cline { 2 - 4 } & Promedio & Mínimo & Máximo \\
\hline 10.000 & 6,38 & 3,82 & 8,34 \\
\hline 20.000 & 4,74 & 3,23 & 6,63 \\
\hline
\end{tabular}

El análisis de la varianza resultó no significativo $(p=0,38)$, por lo cual no hay evidencia experimental de que la densidad de plantación tenga influencia en la producción de biomasa en Eucalyptus camaldulensis. 


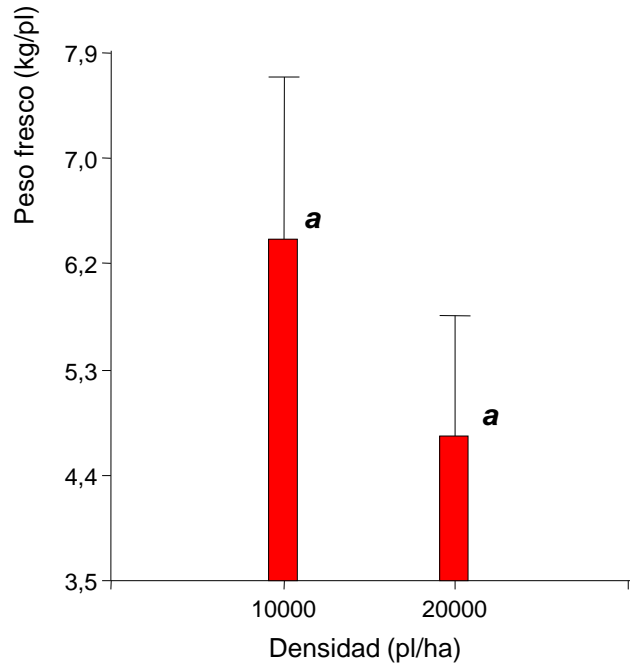

Figura $\mathrm{N}^{\circ} 3$

PESO FRESCO DE BIOMASA SEGÚN DENSIDAD DE PLANTACIÓN

\section{Peso Seco}

El porcentaje de materia seca, respecto de materia en verde (\% peso a peso), determinado para la biomasa cosechada fue de $49,60 \%$. A partir de este dato se estimó el peso seco de cada planta y posteriormente, teniendo en cuenta la densidad de plantación, el peso seco de biomasa por hectárea y por año.

Cuadro $\mathrm{N}^{\circ} 2$

PESO SECO PROMEDIO, MÍNIMO Y MÁXIMO SEGÚN DENSIDAD

\begin{tabular}{|c|c|c|c|}
\hline \multirow{2}{*}{$\begin{array}{c}\text { Densidad } \\
\text { (plantas/ha) }\end{array}$} & \multicolumn{3}{|c|}{$\begin{array}{c}\text { Peso Seco por Planta } \\
\text { (kg) }\end{array}$} \\
\cline { 2 - 4 } & Promedio & Mínimo & Máximo \\
\hline 10.000 & 3,175 & 1,894 & 4,135 \\
\hline 20.000 & 2,366 & 1,735 & 3,521 \\
\hline & \multicolumn{3}{|c|}{ Peso Seco por Hectárea por Año } \\
(kg/ha/año)
\end{tabular}

\section{DISCUSIÓN}

En primer lugar se puede mencionar que el porcentaje de materia seca respecto de la biomasa en fresco obtenido $(49,60 \%)$ es levemente superior al determinado por Forrest y Moore (2008) de 46,78\% en fustes de un año para la misma especie.

Las producciones de biomasa seca de 15,87 t/ha/año (toneladas por hectárea y por año) y 23,66 t/ha/año, en densidades de 10.000 plantas/ha y 20.000 plantas/ha, respectivamente, son 
muy superiores a las 11 t/ha a 1 año de edad hasta las 153 t/ha a los 9 años de edad producidas por Eucalyptus globulus en Etiopía (Zewdie et al., 2009) y a las 16,2 t/ha y 22,2 t/ha (peso por árbol de 0,2 a $32,1 \mathrm{~kg}$ y de 0,3 a $31,1 \mathrm{~kg}$, respectivamente) de materia seca a los tres años, con Eucalyptus globulus y Eucalyptus occidentalis, en la región semiárida de Australia, citadas por Sochacki et al. (2007).

Resultan algo mayores a las mencionadas por Forrest y Moore (2008) en Eucalyptus gunnii, con cosechas anuales durante 14 años y rendimientos de 12,59 a 15,4t/ha/año y por Purse y Richardson (2001), que con Eucalyptus nitens, Eucalyptus gunnii e híbrido de Eucalyptus gunnii $x$ Eucalyptus dalrympleana, estiman producciones de 10 a $15 \mathrm{t} / \mathrm{ha} / \mathrm{año}$, en turnos de corta sugeridos de 8 a 10 años

Valores similares de producción (Luger 1999) son mencionados en rotaciones anuales con Eucalyptus globulus (17 t/ha/año) y con Eucalyptus camaldulensis (20,9 t/ha/año), y otros obtenidos en Grecia con Eucalyptus camaldulensis, con riego y fertilización de 25,6 t/ha/año.

Por último, estos valores son menores a las producciones de $90 \mathrm{t} / \mathrm{ha}$ en Eucalyptus globulus, 65,3 t/ha en Eucalyptus ovata y 49,3 t/ha en Eucalyptus botryoides, regados con agua de efluentes y, turno de corta de 3 años, citados por Guo et al. (2006).

\section{CONCLUSIONES}

El Eucalyptus camaldulensis demuestra ser una especie de buen comportamiento en cultivos de alta densidad de plantación y con cortos turnos de cosecha, regados con aguas provenientes de efluentes domiciliarios. Este recurso hídrico es apto para el riego de este tipo de cultivos, posibilitando el establecimiento, mantenimiento y desarrollo del mismo.

No se observó diferencias estadísticamente significativas en el peso promedio de las plantas respecto de las dos densidades evaluadas.

En el tiempo de implantación del ensayo no se observó la presencia de plagas o enfermedades sobre las plantas.

\section{RECONOCIMIENTOS}

Los autores agradecen a la empresa Cuyoplacas SA, que ha cedido el terreno en donde está instalado el ensayo, y a A la Secretaría de Ciencia, Técnica y Posgrado de la UNCuyo, al Instituto de Energía de la Universidad Nacional de Cuyo y a la empresa YPF SA que han financiado la investigación.

\section{REFERENCIAS}

Arreghini, R.; Bustamante, J. y Paladini, E., 1997. Comportamiento en vivero de eucaliptos australianos en Mendoza. Actas del II Congreso Forestal Argentino y Latinoamericano. Posadas, Misiones.

Calderón, A. y Bustamante, J., 2003. Comportamiento de Eucalyptus spp. en Mendoza, (Argentina) Revista de la Facultad de Ciencias Agrarias, U.N. de Cuyo, Tomo XXXV № 2. 45-52.

Forrest, M. and Moore, T., 2008. Eucalyptus gunnii: A possible source of bioenergy? Biomass and bioenergy 32 978-980.

Guo, L.; Sims, R. and Horne, D., 2006. Biomass production and nutrient cycling in Eucalyptus short rotation energy forests in New Zeland: II. Litter fall and nutrient return. Biomass and bioenergy 30 393-404.

Gutierrez, I. y Ares, A., 1993. Frecuencia de riego en álamos en el valle inferior del Río Colorado provincia de Buenos Aires. Congreso Forestal Argentino y Latinoamericano. Paraná, Entre Ríos. 
Luger, E., 1999. Eucalypt - Introduction as energy crop.

Disponible en http://www.blt.bmlf.gv.at/vero/veroeff/0797_Eucalypt_introduction_e.pdf (Consultada 16/08/2010)

Purse, J. G. and Richardson, K., 2001. Short rotation single stem tree crops for energy in the UK- an examination with Eucalyptus. Aspects of applied Biology № 65. Biomass and Energy Crops II. 13-19.

Riu, N. y Settepani V., 2004. Requerimiento hídrico álamos de 6 años en Rivadavia, Mendoza (Argentina) Revista de la Facultad de Ciencias Agrarias, UNCuyo, Tomo XXXVI, №1, 63-68.

Senelwa, K. and Sims, R., 1999. Fuel characteristics of short rotation forest biomass Biomass and bioenergy 17 127-140.

Sochacki, S.; Harper, R. and Smettem, K., 2007. Estimation of woody biomass production from short-rotation bio-energy system in semid-arid Australia. Biomass and bioenergy 31 608-616.

Tubby, I. and Armstrong, A., 2002. Establishment and Management of short rotation coppice. Practice Note , September 2002. Forestry Commission. 12 páginas

Zewdie, M.; Olsson, M. and Verwijst, T., 2009. Above-ground biomass production and allometric relations of Eucalyptus globulus Labill. Coppice plantations along a chronosequence in the central highlands of Etiopia. Biomass and bioenergy 33 421- 428. 